\title{
Immunomodulatory effect of plasmids co-expressing cytokines in classical swine fever virus subunit gp55/E2-DNA vaccination
}

\author{
Daniel WIENHOLD ${ }^{\mathrm{a}}$, Elisenda ARMENGOL ${ }^{\mathrm{a}}$, Annette MARQUARDT ${ }^{\mathrm{a}}$, \\ Christian MARQUARDT ${ }^{\mathrm{a}}$, Heiner VOIGT ${ }^{\mathrm{a}}$, Mathias BÜTTNER ${ }^{\mathrm{a}}$, \\ Armin SAALMÜLLER ${ }^{\mathrm{b}}$, Eberhard PFAFF ${ }^{\mathrm{a} *}$ \\ ${ }^{a}$ Friedrich Loeffler Institute, Institute of Immunology, Paul-Ehrlich-Strasse 28, 72076 Tübingen, \\ Federal Republic of Germany \\ ${ }^{\mathrm{b}}$ Clinical Immunology, University of Veterinary Medicine Vienna, Veterinärplatz 1,
} 1210 Wien, Austria

(Received 28 April 2004; accepted 2 December 2004)

\begin{abstract}
The aim of this study was to determine the immunomodulatory effects of IL-12, IL-18 and CD154 (CD40 ligand, CD40L) in DNA-vaccination against the classical swine fever virus. Four recombinant plasmids were constructed including the CSFV coding region for the glycoprotein gp55/E2 alone or together with porcine IL-12, IL-18 or CD154 genes. Five groups of four pigs each were immunized intramuscularly (i.m.) three times with the respective constructs. The control group was inoculated with empty plasmid DNA. Eighteen days after the final immunization, the pigs were challenged with a lethal dose of CSFV strain Eystrup and monitored for a further 16 days. This study showed that co-delivery of IL-18 and CD154 induced an earlier appearance of serum antibodies, reduced B-cell deficiency after infection and protected pigs against a lethal CSFV infection. In contrast, co-delivery of IL-12 led to a reduced titer of neutralizing antibodies and protection against a lethal CSFV challenge in comparison to the other pigs and to pigs that were immunized with a gp55/E2 plasmid alone.
\end{abstract}

DNA vaccination / classical swine fever virus

\section{INTRODUCTION}

Classical swine fever virus (CSFV) is a member of the genus Pestivirus within the Flaviviridae and causes a highly infectious and fatal disease in pigs. Infection of swine with classical swine fever virus is characterized by fever, haemorrhages and mortality [9]. In several European countries, out- breaks of CSFV occur periodically in domestic pigs and are always combined with high economic losses [27]. Although highly efficacious, the use of conventional, attenuated live virus CSFV-vaccines do not allow a distinction between naturally infected and vaccinated pigs, therefore the European Union (EU) currently pursues a non-vaccination policy including the stamping-out of

\footnotetext{
* Corresponding author: eberhard.pfaff@tue.bfav.de
} 
infected herds. Marker vaccines based on recombinant glycoprotein E2 allow discrimination between vaccinated and infected animals. Several groups working on CSFV vaccines have shown that especially the E2protein is able to induce a specific and protective immune response against CSFV after vaccination with an E2-expressing recombinant virus $[17,22,32]$, by immunization with baculovirus expressed E2 protein $[5,44,45]$. These experiments have demonstrated that glycoprotein E2 is the most immunogenic and protective protein of CSFV inducing e.g. a high neutralizing antibody titer $[1,45]$.

Recombinant baculovirus expressed E2glycoprotein vaccines have been on the market for several years and are somewhat effective, however they are not able to inhibit virus spread from infected animals [12]. Therefore the efficacy of the vaccine is incomplete.

Another approach for more effective vaccines against CSFV infection is based on DNA-vaccines [1]. Vaccination with E2-DNA allows the induction of a long term immune response and as mentioned above the serological differentiation between vaccinated and infected animals [44]. Nevertheless the immunogenicity of the DNAconstructs is sometimes low and the immunostimulatory capacity might be improved by other components like cytokine genes or CpG-motifs [4, 20, 40].

For other species, it has been previously described that co-administration of cytokine genes with DNA-vaccines increases the immune response and modifies the immune response towards a Th1- or Th2-type reaction $[4,14,20,38]$. In various infections the Th1 or Th2 differentiation of helper T lymphocytes is pivotal to lead to a protective immune response.

In order to enhance the immunostimulatory effect of an E2-DNA vaccine and to direct the E2-specific immune response towards a protective immunity, we used 3 different genes of proteins involved in the onset and control of an immune response: genes of two cytokines, IL-12, IL-18 and the sequence of a regulatory cell surface molecule; CD154 or CD40L. IL-12 is a heterodimeric molecule composed of the covalently linked subunits p35 and p40. It is secreted by peripheral B- and T-cells after induction through bacteria, bacterial products, and parasites [10] as well as by dendritic cells [19]. IL-12 induces a strong IFN- $\gamma$ expression in NK cells $[24,49]$ and promotes a Th1 response under most experimental conditions $[4,38]$. Other cytokines such as IL-18, a monomeric molecule, which is expressed as a $24 \mathrm{kDa}$ precursor protein [28] and after being processed by the IL-1- $\beta$ converting enzyme, are able to stimulate both the Th1 and the Th2 immune response depending on the surrounding cytokine milieu [29]. Finally, CD154 (CD $40 \mathrm{~L}$ ) as a type 2 transmembrane protein has been reported to be expressed on activated $\mathrm{T}$ - and B-cells as well as on natural killerand dendritic cells $[8,30]$. The CD40LCD40 interaction influences and sustains co-stimulatory signaling in APC which is essential for T-cell dependent antibody response [3, 16]. The CD154 activation of immune cell populations is mainly classified as the Th2-response, depending on the immunological microenvironment.

For a better understanding of the porcine immune response against CSFV, the effect of co-delivery of the porcine immunomodulating genes on the efficacy of a CSFV E2DNA vaccine has been tested in domestic swine in a stringent CSFV challenge trial.

\section{MATERIALS AND METHODS}

\subsection{Cloning and DNA preparation}

Classical swine fever virus glycoprotein gp55/E2 (U45478) was isolated and cloned (HindIII/NotI) into the eucaryotic plasmid expression vector pcDNA4HisMax using standard protocols.

For subcloning of the cytokine genes, additional restriction sites (Kpn I/BamHI/ 
NotI/XhoI/NruI/StuI) were inserted into the pcDNA3 plasmid KpnI/StuI. The genes encoding porcine IL-18 (U68701) and CD154 [48] were first isolated using primers including KpnI/NotI restriction sites and subcloned in the modified pcDNA3 plasmid. The IL-12 p35 and p40 subunit genes were isolated as described previously [15]. To allow the expression of the heterodimeric IL-12 protein, the p40 subunit was linked to an IRES sequence from the pIRES1neo plasmid (Clontech, Palo Alto, USA) followed by the p35 subunit and was also subcloned (BamHI/SalI ligated to XhoI) in the modified pcDNA3 plasmid. A second separate transcription unit including the CMV promotor and the cytokine genes was isolated from the modified pcDNA3 plasmid after a NruI digestion. Completing the second transcription units were ligated to the cleaved (PmlI) pcDNA4-E2 plasmid. The gene sequences were verified by the dideoxy nucleotide chain termination method using the BigDye Terminator Cycle Sequencing v2.0 Kit (Applied Biosystems, Foster, USA) and were analyzed by the University of Wisconsin Genetics Computer Group (UWGCG) Software (Accelrys, Munich, Germany) [11]. The plasmid DNA was purified by caesium chloride centrifugation for vaccination in pigs.

\subsection{Transfection and expression}

To check the transient expression of porcine CD154 and the classical swine fever virus glycoprotein gp55/E2, $5 \times 10^{5}$ porcine MAX-cells [31] were seeded and incubated for $24 \mathrm{~h}$. The MAX-cells were transfected using $1 \mu \mathrm{g}$ DNA and Effecten ${ }^{\circledR}$ (QIAGEN, Hilden, Germany) according to the manufacturer's protocol. Twenty-four hours after transfection, the cells were analyzed by immunofluorescence for CD154 and gp55/ E2 expression using specific monoclonal antibodies. For the immunofluorescence staining, transfected cell monolayers were washed once with PBS and fixed with 100\% $\mathrm{EtOH}$ for $15 \mathrm{~min}$ at room temperature for the detection of CD154 expression or $20 \mathrm{~min}$ with 3\% formaldehyde including 10\% Triton $\mathrm{X}$ for the analysis of the E2 expression. After fixation, the cells were washed three times with PBS and incubated either with a mouse monoclonal anti-human CD154 (5C8, ATCC, Manassas, VA, USA) or with the mouse monoclonal anti-E2 antibody a18 [46] for $1 \mathrm{~h}$ at room temperature. After incubation, the cells were washed three times with PBS and stained with propidium iodide. The binding of the CD154 or E2 specific antibodies was visualized with fluorescein-isothiocyanate (FITC)-conjugated anti-mouse IgG antibodies (Dianova, Hamburg, Germany).

For the detection of the expressed porcine IL-12 and IL-18, the supernatant of transiently transfected MAX-cells was collected $24 \mathrm{~h}$ after transfection. Since there was a lack of specific monoclonal antibodies against porcine IL-12 and IL-18, their production was analyzed using their biological activity to enhance the cytolytic activity of natural killer (NK) cells. Assays for the detection of the activity of natural killer cells were performed as described [7, 36]. In brief, K562 tumor cells were labeled with $\mathrm{Na}^{51} \mathrm{CrO}_{4}$ (Amersham, Braunschweig, Germany) for $90 \mathrm{~min}\left(100 \mu \mathrm{Ci} / 10^{6}\right.$ cells $)$, washed and incubated for $16 \mathrm{~h}$ along with freshly isolated effectors (PBMC) at an effector-to-target cell ratio of 100:1. The percentage of specific cytolytic activity was calculated as described previously [35].

\subsection{Vaccination experiment and challenge}

Five groups of four 6 week-old conventional pigs were each immunized intramuscularly (i.m.) with $1000 \mu \mathrm{g}$ of the respective plasmids. All pigs received two boost immunizations $(1000 \mu \mathrm{g})$ on day 22 and 44 post vaccination (p.v.). 18 days after the last immunization, the pigs were challenged with a lethal dose of $10^{5} \mathrm{TCID}_{50}$ of CSFV strain Eystrup [26]. The pigs were bled every week before and on days 4, 7, 10 and 14 after challenge. Besides monitoring the 
clinical signs, the pigs were controlled daily by measuring rectal body temperature. Possible viraemia and virus excretion was tested on days 4, 7,10 and 14 after challenge by collecting blood samples and nasal swabs as well as organ samples on the day of slaughter.

\subsection{Antibody response}

All sera were tested in an ELISA for E2specific antibodies $\left(\right.$ Chekit $^{\circledR}$, Bommeli Diagnostics, Bern, Switzerland) and in a neutralization peroxidase-linked assay (NPLA) [41] for the titer of CSFV-specific neutralizing antibodies. NPLA titers are expressed as the reciprocal of the serum dilution that neutralizes 100 TCID $_{50}$ of CSFV strain Glentorf in 50\% of the replicate culture.

\subsection{Virus isolation}

The collected blood, organ samples and nasal swabs of the pigs were quantitatively tested for the presence of viable virus $\left(\mathrm{TCID}_{50}\right)$ using an immuno-peroxidase monolayer assay (IPMA) as described [47]. Virus titers were calculated as $\log 10 \mathrm{TCID}_{50} / \mathrm{mL}$.

\subsection{Isolation of PBMC and FACS analyses}

Peripheral blood mononuclear cells (PBMC) were separated from venous blood using density gradient centrifugation with Ficoll-Hypaque (PAA, Linz, Austria) [34].

Staining of PBMC for flow cytometric analyses was performed by incubation with a monoclonal antibody against human CD21 showing reactivity with the porcine CD21 (B-ly4, IgG1, BD Pharmingen, San Diego, CA, USA) followed by incubation with an isotype-specific PE-conjugated goat-anti mouse $\operatorname{IgG}_{1}$ antibody (Southern Biotech, Birmingham, AL, USA). All incubation periods were done on ice for $20 \mathrm{~min}$. After each incubation step, the cells were washed twice with FACS buffer (PBS with- out $\mathrm{Ca}^{2+}$ and $\left.\mathrm{Mg}^{2+}, 2 \% \mathrm{FCS}\right)$. Labeled cells were resuspended in FACS buffers and analyzed in a FACStarplus (BD Biosciences, Mountain View, CA, USA) as described [36]. The percentages of CD21-positive B lymphocytes were calculated using WinMDI or Cell Quest ${ }^{\circledR}$ software (BD Biosciences).

\section{RESULTS}

\subsection{Cloning and in vitro expression experiments}

The gene encoding the full length E2/ gp55 protein from CSFV was isolated and cloned into the expression vector pcDNA4HisMax (Fig. 1A). The isolated and subcloned genes for IL-12, IL-18 and CD154 were additionally inserted into the pcDNA4HisMAX-E2 plasmid using a separate transcription unit (Fig. 1B). The expression of the encoded E2 glycoprotein and the porcine CD40L was confirmed by immunofluorescence staining, after transfection into MAX cells, using a monoclonal anti-E2 antibody for the E2 glycoprotein (Fig. 2A.) and a cross reacting monoclonal anti-human CD40L antibody for the porcine CD40L (Fig. 2B). All tested constructs showed high expression of the E2 glycoprotein after transfection into MAX cells. Even high amounts of CD40L were expressed after transfection with pcDNA4-E2-CD40L into MAX cells.

Because of the lack of specific antibodies against porcine IL-12 and IL18, the expression of both cytokines was tested by their biological activity using a natural killer (NK) assay. The results showing the enhancement of the NK activity are represented in Figures 3 (A) for IL-12 and 3 (B) for IL-18. The figures show a stimulation of NK-activity for the supernatant of pcDNA4HisMAXE2-IL-12 transfected MAX cells in contrast to the supernatant of MAX cells transfected with the pcDNA4HisMAX-E2 plasmid alone, which served as a negative control. 
A

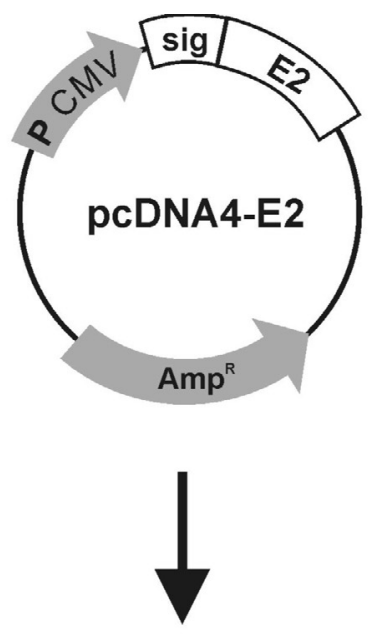

B

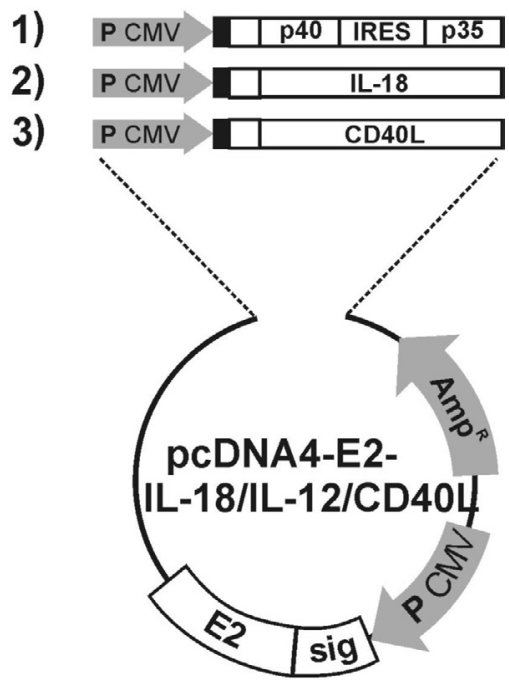

Figure 1. The E2 sequence from CSFV strain "Alfort" was cloned into plasmid pcDNA4 HisMax (A). Additionally, separate transcription units including porcine IL-12 (1), IL-18 (2) and the CD40L (3) under control of a CMV (cytomegalovirus) promoter were cloned into the pcDNA-E2 plasmid (B).

The increase of the NK activity by the supernatant of pcDNA4HisMAX-E2-IL12 transfected MAX cells is comparable with the effect of the adenoviral recom- binant porcine IL-12 supernatant which served in all assays as a positive control.

Comparable to the effect of IL-12, we also detected an enhancement of NK activity by adding the supernatant of pcDNA4HisMAX-E2-IL-18 transfected MAX cells to the NK cells. As mentioned above, the pcDNA4HisMAX-E2 plasmid served as the control. With these results, we were able to demonstrate in a cell based assay the biological activity of the pcDNA4-E2-IL-12 and pcDNA4-E2-IL-18 plasmids, which were thereafter used in the in vivo immunization experiments.

\subsection{CSFV antibody response in DNA vaccinated pigs}

Previous reports about vaccination trials against CSFV present a correlation between humoral immune response and protection. Therefore, we decided to monitor the success of the DNA-immunization with the respective plasmids and the influence of the addition of the immuno-regulatory molecules IL-12, IL-18, and CD154 in a first step by the development of CSFV-specific neutralizing antibodies after vaccination. All pigs received a single immunization and two boost immunizations in a 22 day-interval. The onset and the magnitude of the humoral immune response illustrated by the appearance and the titer of CSFV-specific neutralizing antibodies is presented in Table I. The animals in group No.1 were immunized with naked pcDNA4HisMAX plasmid DNA and served as positive controls for the virus challenge. As expected, none of the animals showed a detectable CSFV-E2-specific antibody titer after vaccination and the time after the challenge infection was too short for the development of virus-specific antibodies. All control animals had to be slaughtered 6-7 days after the challenge infection. Group 2 animals were immunized with the pcDNA4HisMAXE2 plasmid. The animals showed heterogeneous antibody titers after vaccination. In one swine, the antibody titer was very low, 


\section{A}

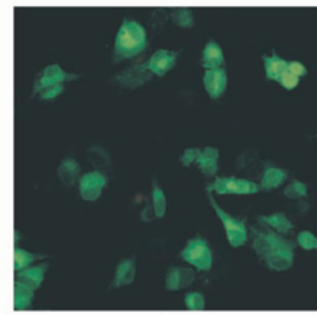

1

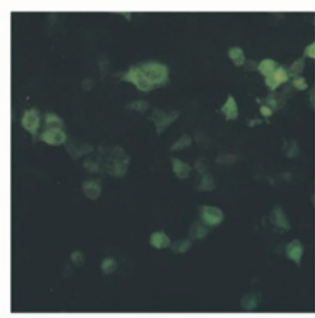

4

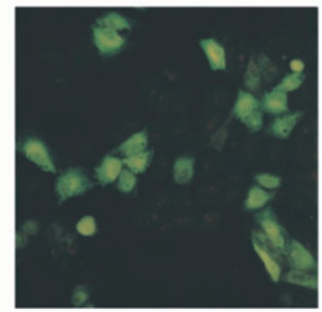

2

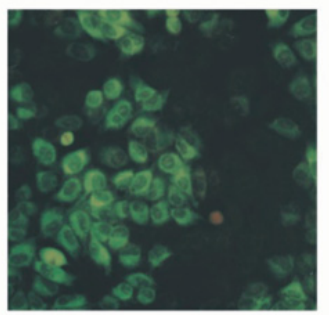

5

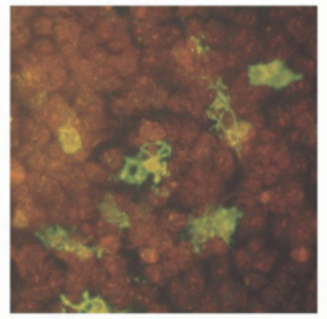

7

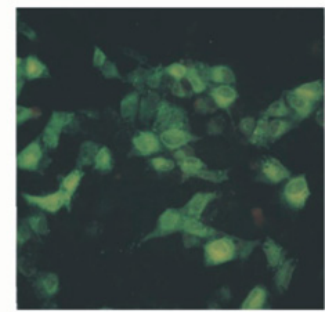

3

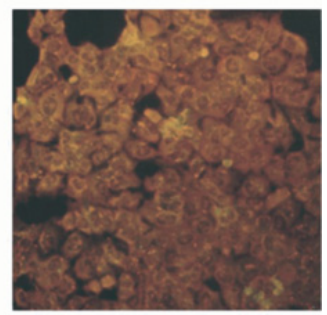

6

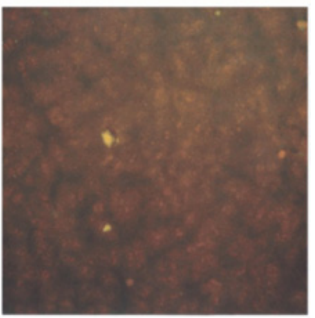

8

Figure 2. Immunofluorescence analysis. (A) The porcine MAX cell line was transfected with the pcDNA4-E2 (1), pcDNA4-E2-IL12 (2), pcDNA4-E2-IL-18 (3), pcDNA4-E2-CD40L (4) plasmid. Transfected as well as CSFV(MOI 1) infected (5) MAX cells showed a distinct green fluorescent granula after incubation with a mouse monoclonal anti-E2 antibody. No green fluorescent granula was detected after incubation of native MAX-cells (6). (B) The porcine MAX cell line shows a distinct green fluorescent granula after transfection with the pcDNA4-E2-CD40L plasmid (7) and incubation with a monoclonal anti human CD40L antibody. No green fluorescent granula was detected after incubation of native MAX-cells (8). The nuclei of the propidium iodide stained cells are colored in orange-red (for a colour version of this figure see www.edpsciences.org/vetres).

whereas three out of four pigs developed CSFV-specific neutralizing antibodies with a reciprocal serum dilution of $60-1600$ for neutralizing antibodies before challenge infec- tion against $100 \mathrm{TCID}_{50}$ of CSFV strain Glentorf in the neutralizing peroxidaselinked antibody assay (NPLA). All pigs showed an increase of antibody titers after 
A

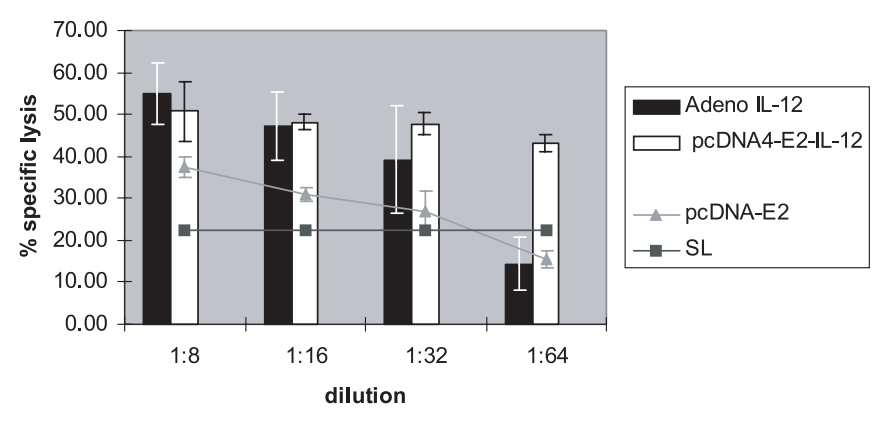

B

\section{IL-18}

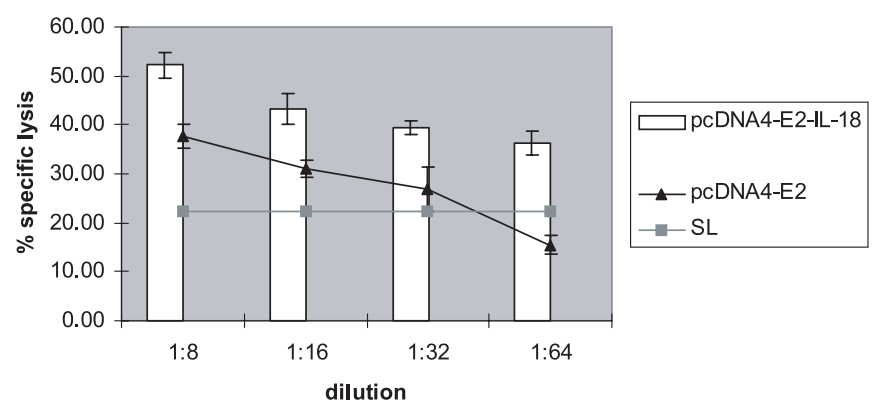

Figure 3. Dose-depended enhancement of NK activity by IL-12 and IL-18. PBMC were incubated with medium (sl) or different concentrations (1:1- 1:64) of supernatant from pcDNA4-E2-IL12 (A), or pcDNA4-E2-IL-18 (B) transfected MAX cells. The specific lysis of K562 tumor cells was calculated as described in the Materials and methods. Supernatants form MAX cells transfected with pcDNA4-E2 and from cells infected with a recombinant IL-12 adenovirus (Ad-IL-12) served as controls.

challenge infection. Two pigs developed up to $>1600$ antibody titers ten days postinfection. The animal with the low titer of neutralizing antibodies prior to the infection kept the low antibody titer and showed only an increase of a reciprocal NPLA-titer of 256 on 14 dpi. One other animal (animal No. 4) died under sample collection on day 7 post infection.

The antibody response of group 3, which included pigs receiving the pcDNA4HisMAX-
E2-IL-12 plasmid, was poor. Only one of four pigs had a detectable NPLA-titer before challenge infection and only two pigs showed a detectable antibody response after challenge. Two pigs, which were not protected by the vaccination had to be euthanized 6-7 days after challenge infection without measurable antibody titers. In contrast, in group 4, treated with the pcDNA4HisMax-E2-IL-18 plasmid (Tab. I, group 4) three out of four animals developed a 
Table I. NPLA (neutralizing peroxidase-linked antibody assay) results are expressed as the reciprocal of the serum dilution neutralizing $100 \mathrm{TCID}_{50}$ of CSFV strain Glentorf in $50 \%$ of the replicate culture. The time points of serum sampling after challenge infection (day 61) are indicated in bold.

\begin{tabular}{|c|c|c|c|c|c|c|c|c|c|c|c|c|}
\hline Plasmid & $\begin{array}{c}\text { Group } \\
\text { No. }\end{array}$ & $\begin{array}{c}\text { Animal } \\
\text { No. }\end{array}$ & Day 29 & day 36 & Day 43 & Day 50 & Day 57 & Day 65 & Day 68 & Day 71 & Day 75 & Day 81 \\
\hline \multirow[t]{2}{*}{ pcDNA4HisMax } & 1 & 1 & - & - & - & - & - & - & - & & & \\
\hline & & 2 & - & - & - & - & - & - & - & & & \\
\hline \multirow[t]{4}{*}{ pcDNA4HisMax-E2 } & 2 & 3 & - & - & - & - & 64 & 192 & 320 & $>2.5 \times 10^{4}$ & $>2.5 \times 10^{4}$ & $>2.5 \times 10^{4}$ \\
\hline & & 4 & - & 60 & n.d & 60 & 60 & 120 & 240 & & & \\
\hline & & 5 & - & 300 & 300 & 800 & 1600 & n.d. & 800 & 1600 & $>2.5 \times 10^{4}$ & $>2.5 \times 10^{4}$ \\
\hline & & 6 & - & - & - & 2 & 4 & 3 & 4 & 6 & 256 & \\
\hline \multirow{4}{*}{$\begin{array}{l}\text { pcDNA4HisMax- } \\
\text { E2-IL-12 }\end{array}$} & 3 & 7 & - & - & n.d. & - & - & - & & & & \\
\hline & & 8 & - & - & n.d. & - & - & - & 160 & 1920 & 1200 & 2000 \\
\hline & & 9 & - & - & n.d. & - & - & - & - & & & \\
\hline & & 10 & - & - & n.d. & - & 20 & 30 & 1280 & 9600 & 6400 & 8000 \\
\hline \multirow{4}{*}{$\begin{array}{l}\text { pcDNA4HisMax- } \\
\text { E2-IL-18 }\end{array}$} & 4 & 11 & - & - & n.d. & - & 30 & 80 & 2560 & 9600 & 9600 & $2.4 \times 10^{4}$ \\
\hline & & 12 & - & - & n.d. & 40 & 80 & 120 & 1280 & $2.5 \times 10^{4}$ & $2.5 \times 10^{4}$ & $3.2 \times 10^{4}$ \\
\hline & & 13 & - & - & n.d. & 30 & 320 & 480 & 2560 & $1.3 \times 10^{4}$ & $1 \times 10^{4}$ & $1.2 \times 10^{4}$ \\
\hline & & 14 & 60 & 60 & n.d. & 20 & 240 & 320 & 640 & 3200 & $1.3 \times 10^{4}$ & $1.2 \times 10^{4}$ \\
\hline \multirow{4}{*}{$\begin{array}{l}\text { pcDNA4HisMax- } \\
\text { E2-CD40L }\end{array}$} & 5 & 15 & - & - & n.d. & - & 6 & 6 & 120 & 480 & 2400 & 4000 \\
\hline & & 16 & 10 & 15 & n.d. & 30 & 60 & 40 & 160 & $1.3 \times 10^{4}$ & $1.9 \times 10^{4}$ & $3.2 \times 10^{4}$ \\
\hline & & 17 & - & - & n.d. & 6 & 30 & 20 & 1920 & $2.5 \times 10^{4}$ & $1.9 \times 10^{4}$ & $3.2 \times 10^{4}$ \\
\hline & & 18 & - & - & n.d. & 8 & 120 & 160 & 1280 & 9600 & 4800 & $1.6 \times 10^{4}$ \\
\hline
\end{tabular}

detectable titer of neutralizing antibodies (day 50, titer 20-40) 6 days after the second boost immunization. One individual showed a time-delayed antibody response with an equivalent titer one week later. Interestingly, after the first immunization with the plasmid, the behavior of the fourth animal on this group showed a neutralizing antibody titer of $1 / 60$, which might be interpreted as a very effective immunization. As expected, after the challenge infection, the antibody titers increased in all individuals of the E2IL-18 group very soon, so that 7 days after the challenge infection in nearly all animals, neutralizing antibody titers $>1000$ were obvious. A similar behavior was seen in group 5. These swine were immunized with the pcDNA4HisMax-E2-CD40L plasmid. Three of four pigs showed a significant titer 6 days after the second boost immunization and the fourth one week later. But compared to the former group, the titer was slightly diminished. After challenge infection, all members of this group developed high titers of neutralizing antibodies, but compared to the E2-IL-18 group, they were time-delayed. Whereas most of the animals of group 4 showed a titer of about 1/1000, 7 days after 
the challenge infection nearly all of the swine of the E2-CD154 group reached this titer one week later.

\subsection{Temperature and clinical signs after challenge}

For a further comparison of the efficacy of the DNA-vaccine constructs and for a more detailed characterization of the influence of the immunomodulating molecules, a measurement of body temperature and the determination of the magnitude of the CSFV-caused B-cell depletion after challenge infection were included in the analyses. The body temperature of each group was measured daily after challenge. As expected, all pigs of the pcDNA4HisMAXvaccinated control group 1 developed a high increase in body temperature four days after infection as well as typical CSFVcaused clinical signs and were euthanized on days 6-7 after challenge infection (Fig 4a).

Three of four pigs that were immunized with the pcDNA4HisMAX-E2 plasmid (group 2) did not show any increase in their body temperature above $40{ }^{\circ} \mathrm{C}$ only one pig from this group had a temperature of $41^{\circ} \mathrm{C}$ for one day (Fig. 4b). It is noteworthy that the individual with the highest temperature in this group was the animal with the lowest antibody titer presented in Figure 1, so that the low antibody might explain the increase in the body temperature based on an incomplete protection. Interestingly, all pigs were free of other clinical signs. The immunization protocol for group 3, the group treated with the E2-IL-12 vector, failed in the protection assay. Two pigs out of the four individuals in group 3 who received the pcDNA4HisMAX-E2-IL-12 plasmid developed fever over $40{ }^{\circ} \mathrm{C}$ which was accompanied by clinical signs, since there was dullness, weakness, anorexia and cyanosis of the skin. The affected animals had to be euthanized on days 6 and 7 after challenge infection (Fig. 4c). The other two individuals of this group did not show any increase of their body temperatures over $40^{\circ} \mathrm{C}$ and were without pathological findings.
The other groups of animals in the E2IL-18 and the E2-CD154 groups, which showed after vaccination in the NPLA a distinct antibody titer (Tab. I), were all protected after the challenge infection. Three out of four of the pcDNA4HisMax-E2-IL18 plasmid immunized pigs (group 4) showed, during the experiment, a body temperature which did not rise above $40{ }^{\circ} \mathrm{C}$; only one pig showed an increase in its body temperature to $41^{\circ} \mathrm{C}$ for one day. All pigs were free of any clinical signs (Fig. 4d). A similar behavior was observed for group 5 , the group of pigs that was immunized with the pcDNA4HisMax-E2-CD154 plasmid (Fig. 4e). Two pigs did not develop fever over $40{ }^{\circ} \mathrm{C}$. The other individuals of that group showed temperatures above $40{ }^{\circ} \mathrm{C}$ for one and two days respectively. Also in that group, all pigs were free of any other clinical signs.

\subsection{Depletion of CD21-positive B-lymphocytes after challenge infection}

The clinical data presented in the last chapter were supported by analyses of a depletion of the B-cell population after challenge infection. It has to be mentioned that the absolute leukocyte counts were neglected in these experiments and based on the experience in the analyses of CSFVinfected animals. Only the relative percentages of $\mathrm{CD} 21^{+} \mathrm{B}$-cells were used as indicators for the success or failure of the immunization. To determine the effect of the immuno-modulating molecules-IL-12, IL-18 and CD154- on the immune response after vaccination and on the impairment of virus-caused pathogenic effects, the reduction of the relative number of B-lymphocytes in peripheral blood mononuclear cells (PBMC) was analyzed during the course of infection. B-lymphocytes were detected with a monoclonal antibody against CD21. The results are summarized in Table II. As expected, all control pigs (group 1) showed a rapid decrease of CD21 positive B-lymphocytes. One of the animals 
pcDNA4HisMax

a)

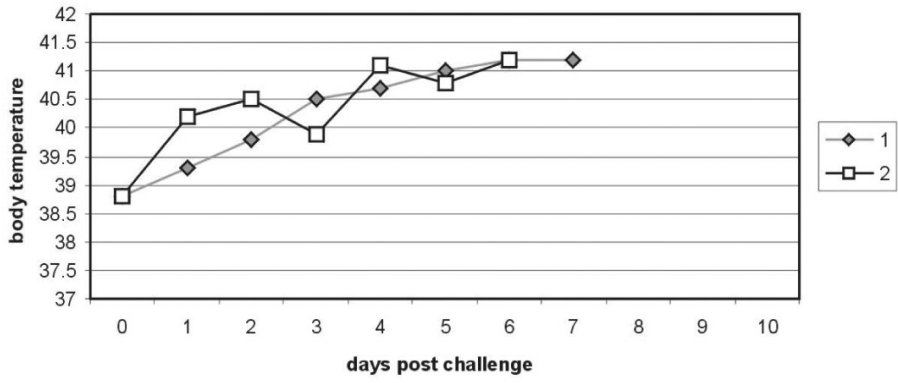

pcDNA4-E2

b)

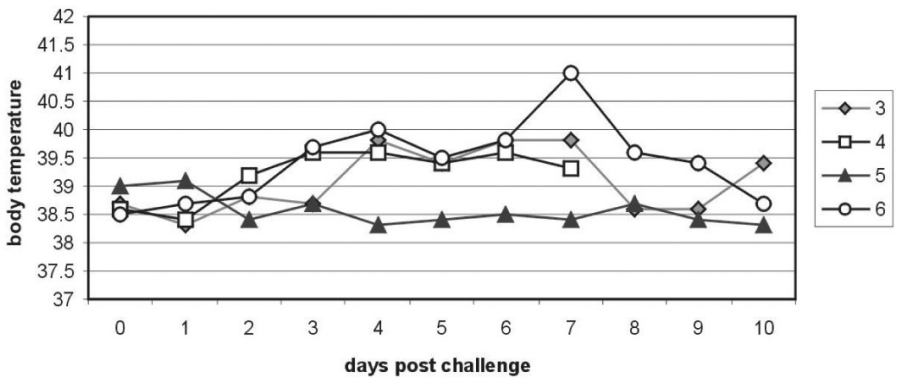

pcDNA-E2-IL12

c)

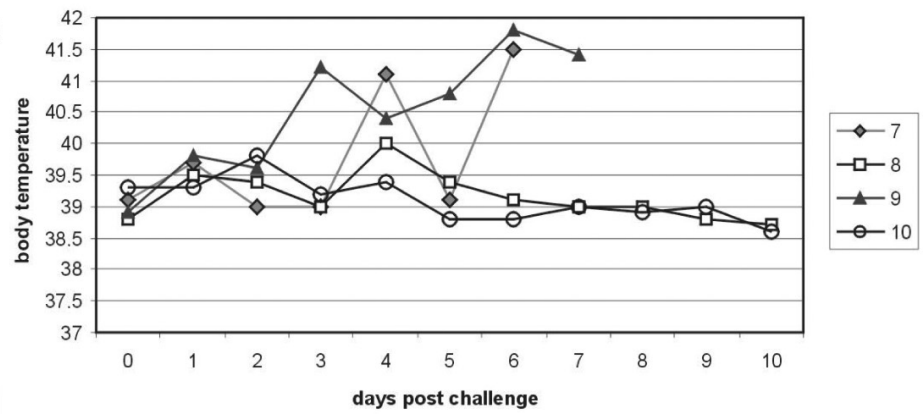

Figure 4. Body temperatures for all pigs were measured rectally every day after challenge infection. Control pigs had to be killed on days 7-8 after challenge. The vaccination plasmid used are indicated in the legend.

showed a reduction after four days post infection from $11 \%$ to $1 \%$. This low percentage of $\mathrm{CD} 21^{+} \mathrm{B}$ lymphocytes stayed stable for several days until the animal had to be euthanized on day 10 p.i. The second individual showed a decrease from $8 \%$ to $3 \%$ after four days p.i. and had to be sacrificed on day 7 p.i. The single member of the E2-vaccinated control group (group 2), which was analyzed during the course of the 

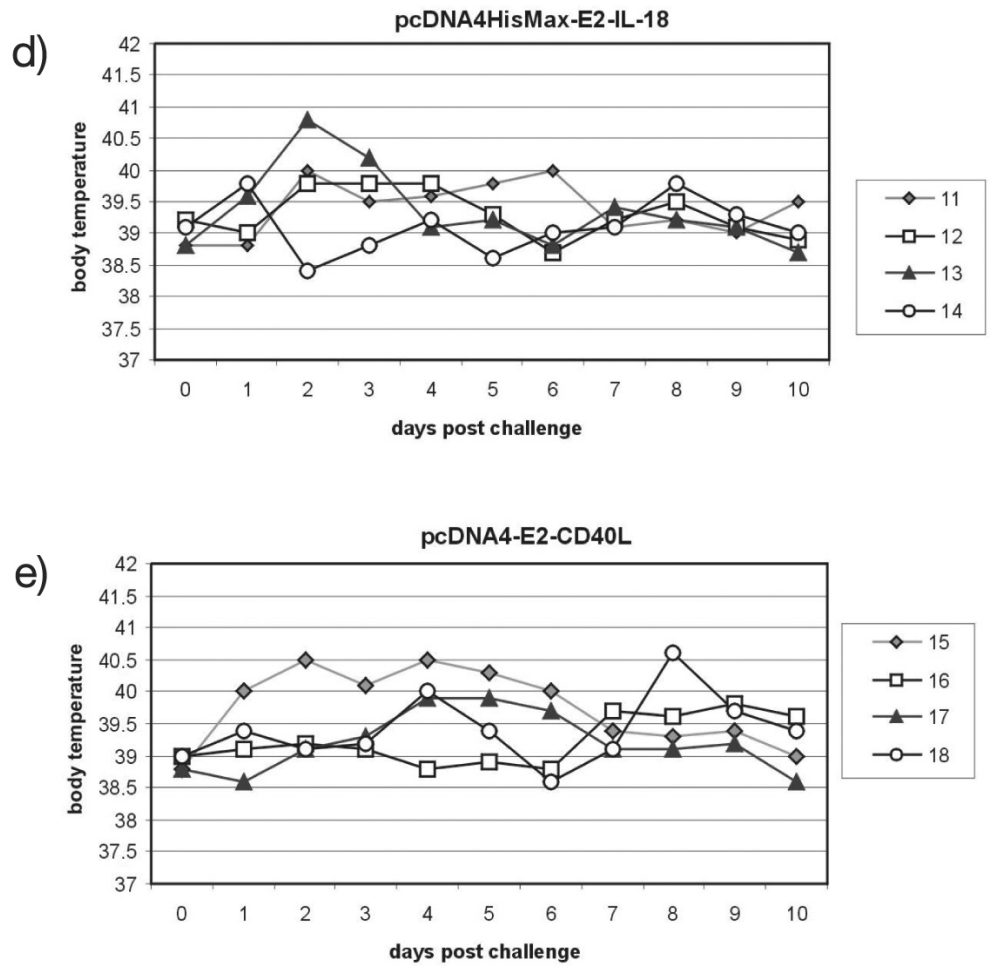

Figure 4. Continued.

infection, indicated a decrease of the relative percentage of CD21-positive cells from $13 \%$ prior to the infection to $6 \%$ on day 4 . Thereafter a low increase to $8 \%$ was detected. This animal died under sample collection on day 7 p.i. Unfortunately, only this single animal from the heterogeneous E2-group was analyzed, therefore we can only speculate about the fate of the B lymphocytes of the other individuals of this group. Group three which was vaccinated with pcDNA4HisMax-E2-IL-12, the IL-12 containing construct, showed a heterogeneous behavior. Two of the four animals demonstrated a severe depletion of $\mathrm{CD} 21^{+} \mathrm{B}$ lymphocytes (9\% to $2 \%$ and $18 \%$ to $1 \%$, respectively) and had to be sacrificed on day 6 as well as on day 7 p.i. This behavior of the animals correlated with the body tem- perature presented in Figure 2. The other two individuals of the group also showed a reduction at the onset of the infection $(5 \%$ to $2 \%$ and $5 \%$ to $3 \%$, respectively) but after 7 days p.i. both animals recovered and the percentage of B-lymphocytes stayed stable during the following days.

The animals vaccinated with the pcDNA4HisMAX-E2-IL18 (group 4) or pcDNA4HisMAX-E2-CD40L construct (group 5) also showed after four days p.i. a weak depression of the percentage of $\mathrm{CD} 21^{+}$ cells, but similar to that of group 4 treated with the IL-18 construct. The percentage of B-lymphocytes also increased continuously in group 5 during the following days.

These results of the relative cell numbers were additionally confirmed by analyses of 
Table II. Percentage of CD21-positive B-lymphocytes in PBMC of pigs after CSFV challenge infection. The cross indicates euthanization of severely affected pigs. In groups 1 and 2 only several animals were tested. The percentage of CD21-positve B lymphocytes was determined as described in the materials and methods.

\begin{tabular}{|c|c|c|c|c|c|c|c|c|}
\hline Plasmid & Group & imal No. & dpi 0 & dpi 4 & dpi 7 & dpi 10 & dpi 14 & dpi 20 \\
\hline \multirow[t]{2}{*}{ pcDNA4HisMax } & 1 & 1 & 11 & 1 & 1 & & & \\
\hline & & 2 & 8 & 3 & & & & \\
\hline pcDNA4HisMax-E2 & 2 & 4 & 13 & 6 & 8 & & & \\
\hline \multirow[t]{4}{*}{ pcDNA4HisMax-E2-IL-12 } & 3 & 7 & 9 & 2 & & & & \\
\hline & & 8 & 5 & 2 & 5 & 7 & 8 & 8 \\
\hline & & 9 & 18 & n.d. & 1 & & & \\
\hline & & 10 & 5 & 3 & 8 & 10 & 10 & 9 \\
\hline \multirow[t]{4}{*}{ pcDNA4HisMax-E2-IL-18 } & 4 & 11 & 7 & 3 & 9 & 13 & 12 & 13 \\
\hline & & 12 & 4 & 3 & 6 & 9 & 10 & 7 \\
\hline & & 13 & 4 & 6 & 6 & 7 & 9 & 9 \\
\hline & & 14 & 6 & 10 & 11 & 9 & 12 & 9 \\
\hline \multirow[t]{4}{*}{ pcDNA4HisMax-E2-CD40L } & 5 & 15 & 5 & 2 & 3 & 6 & 10 & 9 \\
\hline & & 16 & 13 & 11 & 8 & 9 & 16 & 13 \\
\hline & & 17 & 4 & 2 & 4 & 6 & 8 & 7 \\
\hline & & 18 & 6 & 6 & 12 & 17 & 21 & 13 \\
\hline
\end{tabular}

the absolute leukocyte numbers (data not shown).

\subsection{Virus re-isolation after challenge}

Besides the prevention of any clinical signs, a further criterion for the efficacy of a vaccine is its ability to avoid virus replication in the respective tissues and virus spread to neighbor animals. For a quantification of the efficacy, virus titers were determined in the blood, organs (kidney, spleen, mesenteric lymph node, liver, lung, tonsils) and nasal swabs by re-isolation.

As expected, all control animals (group 1) developed viraemia in the blood $\left(\geq 5 \times 10^{3}\right.$ infections virus particles $/ \mathrm{mL}$ ), organs $\left(\geq 5 \times 10^{3}\right.$ virus particles/g) and nasal mucosa $\left(\geq 1 \times 10^{3}\right.$ virus particles $/ \mathrm{mL}$ nasal discharge) (Tab. III).

In group 2 vaccinated with the pcDNA4HisMAX-E2 plasmid, none of the pigs showed any virus in the blood, organs and nasal swabs (Tab. III). This result was different from the result of the pcDNA4HisMAXE2-IL-12 plasmid vaccinated (group 3). Besides the heterogeneities in clinical signs and B-cell depletion, this group was also heterogeneous with regards to viraemia. Virus could be isolated out of the blood from one pig $\left(\geq 5 \times 10^{3}\right.$ virus particles $\left./ \mathrm{mL}\right)$ and in tissue samples from another pig (kidney, spleen, mesenteric lymph node, liver, lung, tonsils $\geq 5 \times 10^{3}$ virus particle/g). No 
Table III. Summary of viraemia in blood, different organs, nasal swab samples and survival from pigs after CSFV infection. Positive tested piglets/ total no. of animals.

\begin{tabular}{|c|c|c|c|c|c|}
\hline \multirow[t]{2}{*}{ Plasmid } & \multirow{2}{*}{ Animal No. } & \multirow{2}{*}{ Survival } & \multicolumn{3}{|c|}{ Viraemia $/ \mathrm{TCID}_{50} / \mathrm{mL}$} \\
\hline & & & Blood & Organs & Nasal swabs \\
\hline \multirow[t]{2}{*}{ pcDNA4HisMax } & 1 & $0 / 2$ & $5 \times 10^{3}$ & $>2.5 \times 10^{6}$ & $1 \times 10^{3}$ \\
\hline & 2 & & $5 \times 10^{4}$ & $>2.5 \times 10^{5}$ & $1 \times 10^{4}$ \\
\hline \multirow[t]{4}{*}{ pcDNA4HisMax-E2 } & 3 & $3 / 4$ & - & - & - \\
\hline & 4 & & - & - & - \\
\hline & 5 & & - & - & - \\
\hline & 6 & & - & - & - \\
\hline \multirow[t]{4}{*}{ pcDNA4HisMax-E2-IL-12 } & 7 & $2 / 4$ & $5 \times 10^{1}$ & - & - \\
\hline & 8 & & - & - & - \\
\hline & 9 & & - & $>5 \times 10^{5}$ & - \\
\hline & 10 & & - & - & - \\
\hline \multirow[t]{4}{*}{ pcDNA4HisMax-E2-IL-18 } & 11 & $4 / 4$ & - & - & - \\
\hline & 12 & & - & - & - \\
\hline & 13 & & - & - & - \\
\hline & 14 & & - & - & - \\
\hline \multirow[t]{4}{*}{ pcDNA4HisMax-E2-CD40L } & 15 & $4 / 4$ & - & - & - \\
\hline & 16 & & - & - & - \\
\hline & 17 & & - & - & - \\
\hline & 18 & & - & - & - \\
\hline
\end{tabular}

virus was detectable in the blood, organs and nasal swabs derived from the two other members of this group (Tab. III). These data clearly correlated with the other clinical parameters and the survival of the individual members of this group.

In group 4, vaccinated with the pcDNA4HisMAX-E2-IL18 plasmid, as well as in group 5, treated with the pcDNA4HisMAXE2-CD40L plasmid, CSFV could not be reisolated in the blood, organs or nasal swabs of any of the individuals (Tab. III).

\section{DISCUSSION}

The glycoprotein E2 is the most immunogenic protein of the classical swine fever virus [6, 13]. It has been previously described that vaccination with the E2 glycoprotein plasmid DNA alone and in a primeboost protocol with a recombinant porcine E2 adenovirus could protect pigs from lethal CSFV infection [18]. However vaccination with an E2 marker vaccine does not inhibit horizontal and vertical virus spread [12]. 
In general cytokines play a primary role in the induction and regulation of immune response against infections [23] and as integrated parts of DNA vaccines, they are able to direct the antigen-specific immune response $[20,25,43]$. In this study we were able to show the effect of three immuno-modulatory molecules in vitro. The NK activity was increased by using the pcDNA4-E2-IL-12 and pcDNA4-E2-IL-18 plasmids in vitro compared to the pcDNA4-E2 plasmid. The stimulating effect of pcDNA-E2 might be explained due to the $\mathrm{CpG}$ motifs in the plasmid backbone [21]. Together with the results from the animal vaccinations, an influence of the cytokines IL-12 and IL-18 and the co-stimulatory molecule CD154 on the immune response against CSFV was clearly demonstrated. For all constructs used for immunization with the exception of the pcDNA4HisMAX and the pcDNA4-HisMAX-E2-IL-12 expression plasmids, all pigs developed neutralizing antibodies as also described in other pre- and post challenge infections [42, 44].

In detail, vaccination with pcDNA4HisMAX-E2-IL-12 only induced in one animal a low E2 specific neutralizing antibody titer pre-challenge infection. After challenge, two of four pigs showed a prominent correlation between the lack of CSFV neutralizing antibodies, the development of fever and viraemia as well as the presence of CSFV in different organs. The one animal who had developed CSFV neutralizing antibodies survived the challenge infection. In addition, the fourth animal who had not developed neutralizing antibodies also survived similar to the described phenomenon after live vaccination [9]. Although T lymphocytes seem to be involved in the development of a protective immune response against CSFV $[2,31]$ the presence of even low titer neutralizing antibodies is a good prognostic marker [17, 33]. Discussing the Th1/Th2 paradigma described for other species as for swine, our results suggest that the expression of IL-12 in conjunction with the E2 glycoprotein prevented the occurrence of CSFV neutralizing serum antibodies
$[37,38]$ probably by a predominance of cellular response, e.g. of IFN- producing cells [52].

Vaccination with the pcDNA4HisMAXE2-IL-18 and pcDNA4HisMAX-E2-CD40L plasmid was characterized in our experiments by the induction of high CSFV neutralizing antibody titers which were clearly linked to the lack of any clinical signs and the typical fever response as seen in the control animals which all succumbed to the CSFV infection. Sporadic temporary increases of physiological body temperature after CSFV challenge infection was observed in this experiment but also occur after vaccination with E2-DNA vaccine [1] and after vaccination using a recombinant E2-Adenovirus [17].

Furthermore, all vaccinated pigs in group 4 (pcDNA4HisMAX-E2-IL-18) and group 5 (pcDNA4HisMAX-E2-CD154) had no virus detectable in the blood and organ samples. In the end, all animals of the two groups survived the lethal challenge infection, whereas one pig of the E2-control group with a lower neutralizing antibody titer had to be sacrificed due to severe clinical signs.

An additional important feature of virulent CSFV infection is the dramatic depletion of B lymphocytes [26, 39]. In contrast to the animals in other groups, we could detect in the blood of members of groups 4 and 5 vaccinated with pcDNA4HisMAX-E2IL-18 and pcDNA4HisMAX-E2-CD154 plasmid DNA, a prevention of a long term B-lymphocyte decline after CSFV challenge infection. A prevention of severe peripheral B-lymphocyte depression can also be explained by the stimulation of the protective humoral immune response as a function of IL-18 [50, 51] and CD40L [3, 16] co-expression in contrast to $E 2$ alone and co-expression with IL-12.

An important point for an emergency vaccine which would be used during an outbreak is that such a vaccine is able to impede transmission of CSFV from infected to noninfected pigs. 
Therefore excretion of the virus was determined by analyzing nasal swabs. There was no virus detectable in all groups with the exception of the non vaccinated control group (group 1). In our study, virus shedding could be inhibited by all of the DNA constructs used, even by the E2-construct alone. Our data on the suppression of virus excretion by the application of E2 alone are different from the results described by Dewulf et al. [12] who could show that application of E2 alone had no effect on virus spread after a challenge infection. These differences might be explained by the diverse application of E2. Dewulf et al. used recombinant baculovirus-expressed E2 for immunization; we used an E2-DNA plasmid.

In contrast to the results from other studies which failed to induce a protective immunity against CSFV by vaccinating with E2-DNA [1] and recombinant E2-containing adenovirus alone [18], we were able to induce a solid protection against viraemia with the pcDNA4HisMAX-E2-IL-18 and pcDNA4HisMAX-E2-CD40L plasmid. This indeed shows the positive influence of IL-18 and CD154 on immunization with E2, but compared to the data from Andrew et al. [1] this effect could also be caused by our vaccination strategy by immunizing three times before challenge infection.

In conclusion, we can assume that DNAvaccination with E2-IL-12 tends to a stimulation of a Th1 immune response and therefore to a suppression of an antigen specific Th2-type immune response. In contrast, vaccination with E2-IL18 and E2CD40L plasmids which seems to induce a Th2-type immune response protected pigs from a CSFV challenge infection, inhibited the nasal secretion of virus and enhanced the CSFV specific immune response compared to pigs vaccinated with an E2-plasmid alone. These results suggest that co-expression of specific cytokines and co-stimulatory molecules in combination with the E2 glycoprotein can enhance the efficacy of DNA vaccination against CSFV. The protective potency of a single vaccination with a cytokine/E2 combination will be the subject of future investigations.

Taking these data together, we show that vaccination with an IL-18- or CD154- modified E2-containing DNA plasmid led to the improvement of the immune reaction against a CSFV challenge infection. Interestingly, a combination of E2-DNA with IL-12 seemed to reduce the efficacy of the vaccine.

\section{ACKNOWLEDGEMENTS}

We thank U. Csacsko for technical support and W. Kramer for photo documentation.

\section{REFERENCES}

[1] Andrew M.E., Morrissy C.J., Lenghaus C., Oke P.G., Sproat K.W., Hodgson A.L.M., Johnson M.A., Coupar B.E.H., Protection of pigs against classical swine fever with DNA-delivered gp55, Vaccine 18 (2000) 1932-1938.

[2] Armengol E., Wiesmüller K.-H., Wienhold D., Büttner M., Pfaff E., Jung G., Saalmüller A., Identification of T-cell epitopes in the structural and non-structural proteins of classical swine fever virus, J. Gen. Virol. 83 (2002) 551-560.

[3] Banchereau J., Bazan F., Blanchard D., Briere F., Galizzi J.P., van Kooten C., Liu Y.J., Rousset F., Saeland S., The CD40 antigen and its ligand, Annu. Rev. Immunol. 12 (1994) 881-922.

[4] Boretti F.S., Leutenegger C.M., Mislin C., Hofmann-Lehmann R., Konig S., Schroff M., Junghans C., Fehr D., Huettner S.W., Habel A., Flynn J.N., Aubert A., Pedersen N.C., Wittig B., Lutz H., Protection against FIV challenge infection by genetic vaccination using minimalistic DNA constructs for FIV env gene and feline IL-12 expression, AIDS 14 (2000) 1749-1757.

[5] Bouma A., De Smit A.J., De Kluijver E.P., Terpstra C., Moormann R.J., Efficacy and stability of a subunit vaccine based on glycoprotein E2 of classical swine fever virus, Vet. Microbiol. 66 (1999) 101-114.

[6] Bouma A., De Smit A.J., De Jong M.C., De Kluijver E.P., Moormann R.J., Determination of the onset of the herd-immunity induced by the E2 sub-unit vaccine against classical swine fever virus, Vaccine 18 (2000) 1374-1381.

[7] Cho D., Lee W.J., Halloran P.J., Trinchieri G., Kim Y.B., Enhancement of porcine natural killer cell activity by recombinant human and murine IL-12, Cell. Immunol. 172 (1996) 29-34. 
[8] Clarke S.R., The critical role of CD40/CD40L in the CD4-dependent generation of $\mathrm{CD}^{+} \mathrm{T}$ cell immunity, J. Leukoc. Biol. 67 (2000) 607-614.

[9] Dahle J., Liess B., A review on classical swine fever infections in pigs: epizootiology, clinical disease and pathology, Comp. Immunol. Microbiol. Infect. Dis. 15 (1992) 203-211.

[10] D'Andreas A., Rengaraju M., Valiante N.M., Chehimi J., Kubin M., Aste M., Chan S.H., Kobayashi M., Young D., Nickbarg E., Production of natural killer cell stimulatory factor (interleukin 12) by peripheral blood mononuclear cells, J. Exp. Med. 176 (1992) 1387-1398.

[11] Devereux J., Haeberli P., Smithies O., A comprehensive set of sequence analysis programs for the VAX, Nucleic Acid Res. 12 (1984) 387-395.

[12] Dewulf J., Laevens H., Koenen F., Mintien K., de Kruif A., An E2 sub-unit marker vaccine does not prevent horizontal or vertical transmission of classical swine fever virus, Vaccine 20 (2001) 86-91.

[13] Dong X.N., Wie K., Liu Z.Q., Chen Y.H., Candidate peptide vaccine induced protection against classical swine fever virus, Vaccine 21 (2002) 167-173.

[14] Dupre L., Kremer L., Wolowczuk I., Riveau G., Capron A., Locht C., Immunostimulatory effect of IL-18-encoding plasmid in DNA vaccination against murine Schistosoma mansoni infection, Vaccine 19 (2001) 1373-1380.

[15] Foss D.L., Murtaugh M.P., Molecular cloning and mRNA expression of porcine interleukin12, Vet. Immunol. Immunopathol. 57 (1997) 121-134.

[16] Foy T.M., Aruffo A., Bajorath J., Buhlmann J.E., Noelle R.J., Immune regulation by CD40 and its ligand GP39, Annu. Rev. Immunol. 14 (1996) 591-617.

[17] Hammond J.M., McCoy R.J., Jansen E.S., Morriss, C.J., Hodgson A.L., Johnson M.A., Vaccination with a single dose of a recombinant porcine adenovirus expressing the classical swine fever virus gp55 (E2) gene protects pigs against classical swine fever, Vaccine 18 (2000) 1040-1050.

[18] Hammond J.M., Jansen E.S., Morrissy C.J., Goff V.W., Meehan C.G., Lenghaus W.C., Sproat K.W., Andrew M.E., Coupar B.E.H., Johnson M.A., A prime-boost vaccination strategy using naked DNA followed by recombinant porcine adenovirus protects pigs from classical swine fever, Vet. Microbiol. 80 (2001) 101-119.

[19] Heufler C., Koch F., Stanzl U., Topar G., Wysocka M., Trinchieri G., Enk A., Steinman R.M., Romani N., Schuler G., Interleukin-12 is produced by dendritic cells and mediates $\mathrm{T}$ helper 1 development as well as interferon- gamma production by T helper 1 cells, Eur. J Immunol. 26 (1996) 659-68.

[20] Ihata A., Watabe S., Sasaki S., Shirai A., Fukushima J., Hamajima K., Inoue J., Okuda K., Immunomodulatory effect of a plasmid expressing CD40 ligand on DNA vaccination against human immunodeficiency virus type-1, Immunology 98 (1999) 436-442.

[21] Klinman D.M., Yi A.K., Beaucage S.L., Conover J., Krieg A.M., CpG motifs present in bacteria DNA rapidly induce lymphocytes to secrete interleukin 6, interleukin 12, and interferon gamma, Proc. Natl. Acad. Sci. USA 93 (1996) 2879-2883.

[22] König M., Lengsfeld T., Pauly T., Stark R., Thiel H.J., Classical swine fever virus: independent induction of protective immunity by two structural glycoproteins, J. Virol. 69 (1995) 6479-6486.

[23] Kourilsky P., Truffa-Bachi P., Cytokine fields and the polarization of the immune response, Trends Immunol. 22 (2001) 502-509.

[24] Manetti R., Parronchi P., Giudizi M.G., Piccinni M.P., Maggi E., Trinchieri G., Romagnani S., Natural killer cell stimulatory factor (interleukin 12 (IL-12)) induces T helper type 1 (Th1)-specific immune responses and inhibits the development of IL-4-producing Th cells, J. Exp. Med. 177 (1993) 1199-1204.

[25] Matsui M., Moriya O., Akatsuka T., Enhanced induction of hepatitis $\mathrm{C}$ virus-specific cytotoxic $\mathrm{T}$ lymphocytes and protective efficacy in mice by DNA vaccination followed by adenovirus boosting in combination with the interleukin 12 expression plasmid, Vaccine 21 (2003) 1629-1639.

[26] Meyers G., Saalmüller A., Büttner M., Mutations abrogating the RNase activity in glycoprotein $\mathrm{E}(\mathrm{rns})$ of the pestivirus classical swine fever virus lead to virus attenuation, J. Virol. 73 (1999) 10224-10235.

[27] Mintiens K., Deluyker H., Laevens H., Koenen F., Dewulf J., De Kruif A., Descriptive epidemiology of a classical swine fever outbreak in the Limburg Province of Belgium in 1997, J. Vet. Med. B Infect. Dis. Vet. Public Health 48 (2001) 143-149.

[28] Muneta Y., Inumaru S., Shimoji Y., Mori Y., Efficient production of biologically active porcine interleukin- 18 by coexpression with porcine caspase- 1 using a baculovirus expression system, J. Interferon Zytokine Res. 21 (2001) 125-130.

[29] Nakanishi K., Yoshimoto T., Tsutsui H., Okamura H., Interleukin-18 regulates both Th1 and Th2 responses, Annu. Rev. Immunol. 19 (2001) 423-474.

[30] Noelle R.J., Roy M., Sheoherd D.M., Stamenkovic I., Ledbetter J.A., Aruffo A., A $39-\mathrm{kDa}$ protein on activated helper $\mathrm{T}$ cells 
binds CD40 and transduces the signal for cognate activation of B cells, Proc. Natl. Acad. Sci. USA 89 (1992) 6550-6554.

[31] Pauly T., Elbers K., König M., Lengsfeld T., Saalmüller A., Thiel H.J., Classical swine fever virus-specific cytotoxic $\mathrm{T}$ lymphocytes and identification of a T cell epitope, J. Gen. Virol. 76 (1995) 3039-3049.

[32] Peeters B., Bienkowska-Szewczyk K., Hulst M., Gielkens A., Kimman T., Biologically safe, non-transmissible pseudorabies virus vector vaccine protects pigs against both Aujeszky's disease and classical swine fever, J. Gen. Virol. 78 (1997) 3311-3315.

[33] Rümenapf T., Stark R., Meyers G., Thiel H.J., Structural proteins of hog cholera virus expressed by vaccinia virus: further characterization and induction of protective immunity, J. Virol. 65 (1991) 589-597.

[34] Saalmüller A., Jonjic S., Bühring H.-J., Reddehase M.J., Koszinowski U.H., Monoclonal antibodies reactive with swine lymphocytes. II. Detection of an antigen on resting T cells down-regulated after activation, J. Immunol. 138 (1987) 1852-1857.

[35] Saalmüller A., Reddehase M.J., Bühring H.J., Jonjic S., Koszinowski U.H., Simultaneous expression of CD4 and CD8 antigens by a substantial proportion of resting porcine $\mathrm{T}$ lymphocytes, Eur. J. Immunol. 17 (1987) 1297-1301.

[36] Saalmüller A., Hirt W., Maurer S., Weiland E., Discrimination between two subsets of porcine $\mathrm{CD}^{+}$cytolytic $\mathrm{T}$ lymphocytes by the expression of CD5 antigen, Immunology 81 (1994) 578-583.

[37] Schirmbeck R., Reimann J., Modulation of Gene-Gun-Mediated Th2 Immunity to Hepatitis B Surface Antigen by Bacterial CpG Motifs or IL-12, Intervirology 44 (2001) 115-123.

[38] Shan M., Liu K., Fang H., DNA vaccination of the induction of immune responses by codelivery of IL-12 expression vector with hepatitis C structural antigens, Zhonghua Gan Zang Bing Za Zhi 7 (1999) 236-239.

[39] Susa M., König M., Saalmüller A., Reddehase M.J., Thiel H.J., Pathogenesis of classical swine fever: B-lymphocyte deficiency caused by hog cholera virus, J. Virol. 66 (1992) 1171-1175.

[40] Temperton N.J., Quenelle D.C., Lawson K.M., Zuckerman J.N., Kern E.R., Griffiths P.D., Emery V.C., Enhancement of humoral immune responses to a human cytomegalovirus DNA vaccine: adjuvant effects of aluminum phosphate and $\mathrm{CpG}$ oligodeoxynucleotides, J. Med. Virol. 70 (2003) 86-90.

[41] Terpstra C., Bloemraad M., Gielkens A.L., The neutralizing peroxidase-linked assay for detection of antibody against swine fever virus, Vet. Microbiol. 9 (1984) 113-120.
[42] Terpstra C., Wensvoort G., The protective value of vaccine-induced neutralizing antibody titers in swine fever, Vet. Microbiol. 16 (1988) 123-128.

[43] Triccas J.A., Sun L., Palendira U., Britton W.J., Comparative affects of plasmidencoded interleukin 12 and interleukin 18 on the protective efficacy of DNA vaccination against Mycobacterium tuberculosis, Immunol. Cell Biol. 80 (2002) 346-350.

[44] Van Rijn P.A., Bossers A., Wensvoort G., Moormann R.J.M., Classical swine fever virus (CSFV) envelope glycoprotein E2 containing one structural antigenic unit protects pigs from lethal CSFV challenge, J. Gen. Virol. 77 (1996) 2737-2745.

[45] Van Rijn P.A., van Gennip H.G.P., Moormann R.J.M., An experimental marker vaccine and accompanying serological diagnostic test both based on envelope glycoprotein E2 of classical swine fever virus (CSFV), Vaccine 17 (1999) 433-440.

[46] Weiland E., Stark R., Haas B., Rümenapf T., Meyers G., Thiel H.J., Pestivirus glycoprotein which induces neutralizing antibodies forms part of a disulfide-linked heterodimer, J Virol. 64 (1990) 3563-3569.

[47] Wensvoort G., Bloemraad M., Terpstra C., An enzyme immunoassay employing monoclonal antibodies and detecting specifically antibodies to classical swine fever virus, Vet. Microbiol. 17 (1988) 129-140.

[48] Wienhold D., Berger N., Armengol E., Buttner M., Saalmuller A., Pfaff E., Cloning, sequencing and expression of porcine CD40 ligand in Escherichia coli and human and porcine cells, Cytokine 20 (2002) 274-282.

[49] Wu C.Y., Demeure C., Kiniwa M., Gately M., Delespesse G., IL-12 induces the production of IFN-gamma by neonatal human CD4 T cells, J. Immunol. 151 (1993) 1938-1949.

[50] Yoshimoto T., Tsutsui H., Tominaga K., Hoshino K., Okamura H., Akira S., Paul W.E., Nakanishi K., IL-18, although antiallergic when administered with IL-12, stimulates IL-4 and histamine release by basophils, Proc. Natl. Acad. Sci. USA 96 (1999) 13962-13966.

[51] Yoshimoto T., Mizutani H., Tsutsui H., Noben-Trauth N., Yamanaka K., Tanaka M., Izumi S., Okamura H., Paul W.E., Nakanishi K., IL-18 induction of IgE: dependence on $\mathrm{CD}^{+}{ }^{+} \mathrm{T}$ cells, IL-4 and STAT6, Nat. Immunol. 1 (2000) 132-137.

[52] Zuckermann F.A., Husmann R.J., Schwartz R., Brandt J., Mateu de Antonio E., Martin S., Interleukin-12 enhances the virus-specific interferon gamma response of pigs to an inactivated pseudorabies virus vaccine, Vet. Immunol. Immunopathol. 63 (1998) 57-67. 